\title{
Frequency-optimised radio arrays for air-shower detection
}

\section{Aswathi Balagopal V.*1 ${ }^{*}$ Andreas Haungs ${ }^{2}$, Tim Huege ${ }^{2,3}$, Max Renschler ${ }^{1}$, Frank G.} Schröder ${ }^{2,4}$ and Anne Zilles ${ }^{5}$

${ }^{1}$ Institut für Experimentelle Teilchenphysik, Karlsruhe Institute of Technology (KIT), Karlsruhe, Germany

${ }^{2}$ Institut für Kernphysik, Karlsruhe Institute of Technology (KIT), Karlsruhe, Germany

${ }^{3}$ Astrophysical Institute, Vrije Universiteit Brussel, Pleinlaan 2, Brussels, Belgium

${ }^{4}$ Bartol Research Institute and Dept. of Physics and Astronomy, University of Delaware, Newark, DE 19716, USA

${ }^{5}$ Sorbonne Université, CNRS, UMR 7095, Institut d'Astrophysique de Paris, 98 bis bd Arago, 75014 Paris, France

E-mail: aswathi.balagopalekit.edu

\begin{abstract}
The radio detection technique has successfully been applied to the measurement of cosmic-ray induced air showers with energies above $10^{16} \mathrm{eV}$. A majority of these measurements are performed in the frequency band of $30-80 \mathrm{MHz}$, by the existing radio air-shower experiments. Recent results of a simulation study with CoREAS show that the optimisation of the frequency band improves the signal-to-noise ratio. This in turn lowers the energy threshold of detection by an order of magnitude. This talk will focus on the universal application of such a frequency optimisation for all future radio air-shower arrays, which will improve the performance of these future arrays. For this, a simulation study performed for the locations of IceTop, GRAND and the Pierre Auger Observatory using air showers generated by cosmic rays, gamma-rays and neutrinos is utilised.
\end{abstract}

36th International Cosmic Ray Conference -ICRC2019-

July 24th - August 1st, 2019

Madison, WI, U.S.A.

${ }^{*}$ Speaker. 


\section{Introduction}

Since the early 2000's, the field of radio detection of extensive air showers has seen incredible progress. With the help of several radio air-shower experiments, today we understand the radio emission in detail. Most of these experiments using the radio technique operate/operated in the frequency band of 30-80 MHz. So far, the expected emission is seen to be in agreement with all experimental data [1][2]. Also, the existence of a Cherenkov ring where the emission is amplified and coherent up to a few $\mathrm{GHz}$ has been established [3]. With this better understanding of the emission mechanism today, it is natural to revisit the frequency band of operation for future radio arrays.

The motivation for revisiting this problem was derived from the necessity to lower the energy threshold of detection using the radio technique for a future radio array at the South Pole [4], which will be used for the enhancement of IceTop (the surface array of IceCube that measures cosmic ray air showers)[5][6]. This enables the search for PeV energy gamma rays approaching the South Pole from the centre of the Milky Way using the technique of radio detection of air showers [7]. Here, we will discuss the study on the frequency band of operation conducted for the location of IceTop, followed by a similar study done for the locations of the Pierre Auger Observatory and GRAND.

\section{IceTop}

IceTop consists of 162 ice-Cherenkov tanks, grouped into sets of two, forming 81 stations. This particle-detector array cannot measure PeV gamma rays coming from the Galactic Centre, since it always lies at a zenith angle of $61^{\circ}$ at the South Pole. A majority of the particles from these inclined showers do not reach the ground. Hence, we move to the usage of the radio detection technique, since the radio signals are not absorbed in the atmosphere, and can therefore reach the ground. In the simulation study that was focused on this problem, an array of 81 antennas, each lying at the centre of each IceTop station, was used, resulting in a total area of $1 \mathrm{~km}^{2}$ [7] [8] [9]. These air showers were simulated using CoREAS [10].

Since the detection of air showers with the radio technique has been successfully done only for showers with primary energies well above $10^{16} \mathrm{eV}$, the energy threshold needs to be lowered for the detection of PeV energy showers. A major challenge for this is the ability to observe the signal above the level of noise that is seen in radio air-shower experiments. Therefore, the behaviour of the signal and the noise were studied within different frequency bands of operation. Such a comparison between the signal and the noise was done for all the antennas in the array, in order to have an optimal detection in the entire radio array. The noise used for this study includes both Galactic noise, which arises from all the sources within our Galaxy, and thermal noise (from the antenna characteristics and the surroundings). An average behaviour of the Galactic noise was obtained from the Cane model [11] and a constant, conservative level of thermal noise (300 K) was added to this at all frequencies, as described in references [7] and [8]. The typical thermal noise of state-of-the art antennas (SKALA antennas developed for SKA) is $\approx 40 \mathrm{~K}$ within its frequency band of operation [12]. Since these antennas are planned to be used for the IceTop radio enhancement, a thermal-noise case of $40 \mathrm{~K}$ is also considered for the study done for IceTop. 
Figure 1 shows the amplitude of the simulated signals at three different stations in the radio array. These signals are obtained for a shower of zenith angle $61^{\circ}$, produced by a gamma-ray primary with an energy of $10 \mathrm{PeV}$. The three stations are located at the Cherenkov ring (i.e. a station lying at an off-axis angle angle of $1.01^{\circ}$ with respect to the shower maximum at an observation level of $2838 \mathrm{~m}$ above the sea level, giving a distance to the core of $155 \mathrm{~m}$ ), inside the ring (at an off-axis angle of $0.6^{\circ}$, core distance $92 \mathrm{~m}$ ) and outside the Cherenkov ring (at an off-axis angle of $3.16^{\circ}$, core distance $484 \mathrm{~m}$ ). The signals are shown here in the frequency domain. At the Cherenkov ring, the signal has a flat frequency spectrum and is seen to be coherent throughout the frequency range shown here (a few $\mathrm{MHz}$ to a few $\mathrm{GHz}$ ). On the other hand, the signals inside and outside the Cherenkov ring are not coherent in the entire frequency range shown here. The amplitudes of these signals go down at higher frequencies. The fluctuations seen at very high frequencies occur due to thinning artifacts in the simulations.

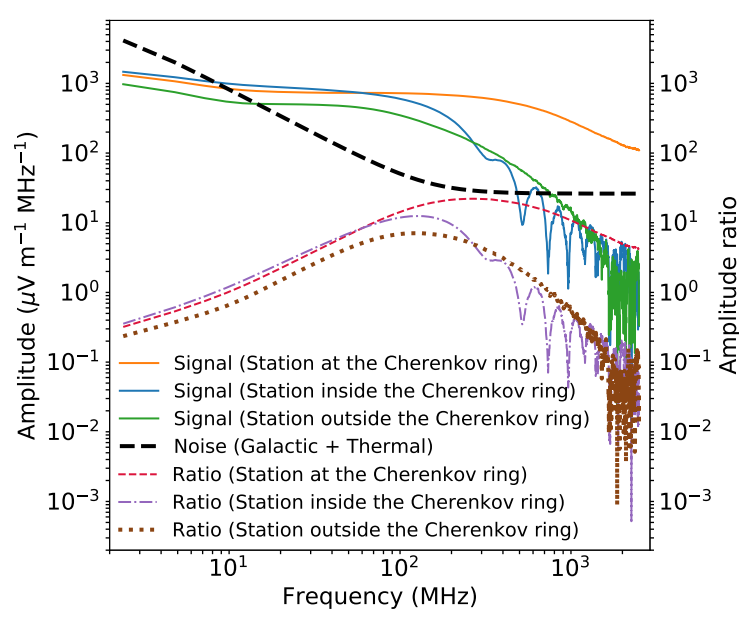

(a) Thermal noise $=300 \mathrm{~K}$

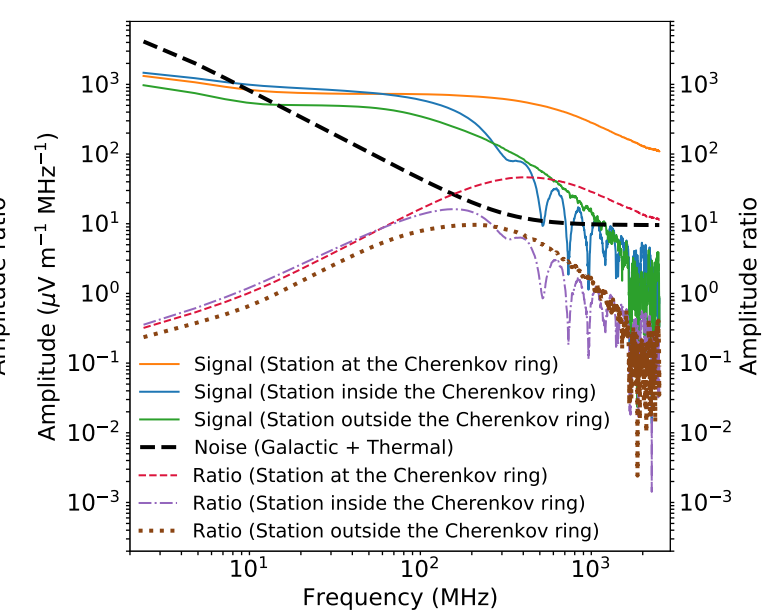

(b) Thermal noise $=40 \mathrm{~K}$

Figure 1: Frequency spectra of signals at three different positions in the shower footprint for a gamma-ray shower with zenith angle $61^{\circ}$ simulated for the location of IceTop. The black dashed curve shows the total noise (Galactic + thermal). The left panel shows the scenario where the thermal noise is $300 \mathrm{~K}$ and the right panel depicts that for a thermal noise of $40 \mathrm{~K}$. Also shown is the ratio of the amplitudes of the signal and the noise at each frequency shown here. Figure adapted from [8].

Also shown in Figure 1 is the amplitude of the noise (with the same normalisation as the signal) in the frequency domain, where a combination of Galactic and thermal noise has been considered, as described above. At lower frequencies the Galactic noise is dominant and at higher frequencies, the constant thermal noise takes over. This results in the initial decreasing nature of the total noise, followed by a flat behaviour at higher frequencies. In Figure 1a, the thermal noise is at a level of $300 \mathrm{~K}$, resulting in a crossover between the Galactic and thermal noise at $\approx 150 \mathrm{MHz}$. Figure $1 \mathrm{~b}$ depicts the scenario when the thermal noise is $40 \mathrm{~K}$, and the cross-over occurs at $\approx 350 \mathrm{MHz}$. The signal also goes down in its amplitude as the frequency increases, especially for the stations inside and outside the Cherenkov ring. Therefore, there is a sweet spot between $100 \mathrm{MHz}$ and 200 $\mathrm{MHz}$, where the noise is going down and the signal is still high enough to give an optimal level of 
signal-to-noise ratio at all the stations shown here, which represent different regions of the radio footprint. To show this more clearly, Figure 1 shows the ratio of the amplitude of the signal and the amplitude of the noise within each frequency bin. This is done for each of the representative stations shown in the figure. Note that this is not the same as the parameter signal-to-noise ratio, which is defined later in this document. The amplitude ratio is shown here only for clarification of the sweet spot seen between $100 \mathrm{MHz}$ and $200 \mathrm{MHz}$. It can be seen that the amplitude ratio is small at lower frequencies due to the higher level of noise. The traditional frequencies between $30 \mathrm{MHz}$ and $80 \mathrm{MHz}$ have lower levels of amplitude ratio than that seen at frequencies between $100 \mathrm{MHz}$ and $200 \mathrm{MHz}$. This is true for the signal at the Cherenkov ring also (Figure 1a). In Figure $1 \mathrm{~b}$ where the thermal noise level is lower, since the flattening of the noise occurs only above $\approx 350 \mathrm{MHz}$, the ratio is high for the station at the Cherenkov ring until this cross-over frequency, after which it starts going down. However, the signals at other regions of the footprint start losing coherence at these higher frequencies. This is especially true for the signal inside the Cherenkov ring. So it still has a higher level of amplitude ratio between $100 \mathrm{MHz}$ and $200 \mathrm{MHz}$. The station outside the Cherenkov ring has a desirable amplitude ratio until $\approx 300 \mathrm{MHz}$.

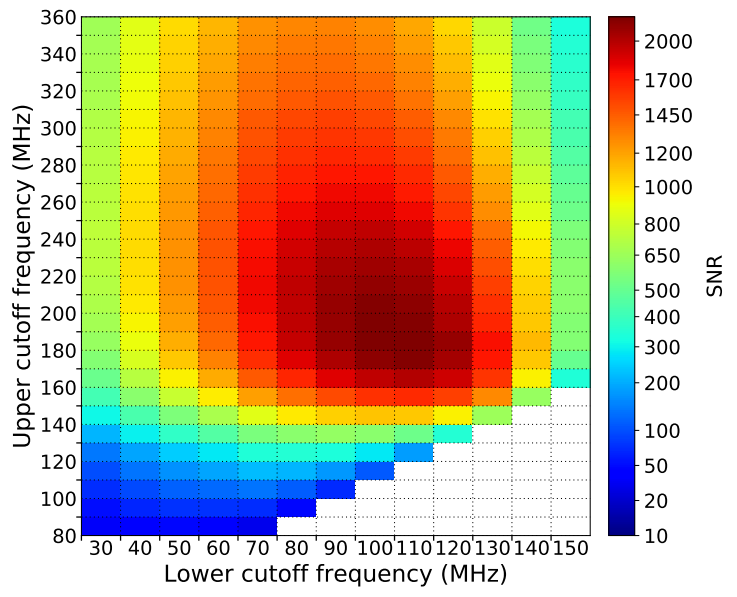

(a) IceTop: Thermal noise $=300 \mathrm{~K}$

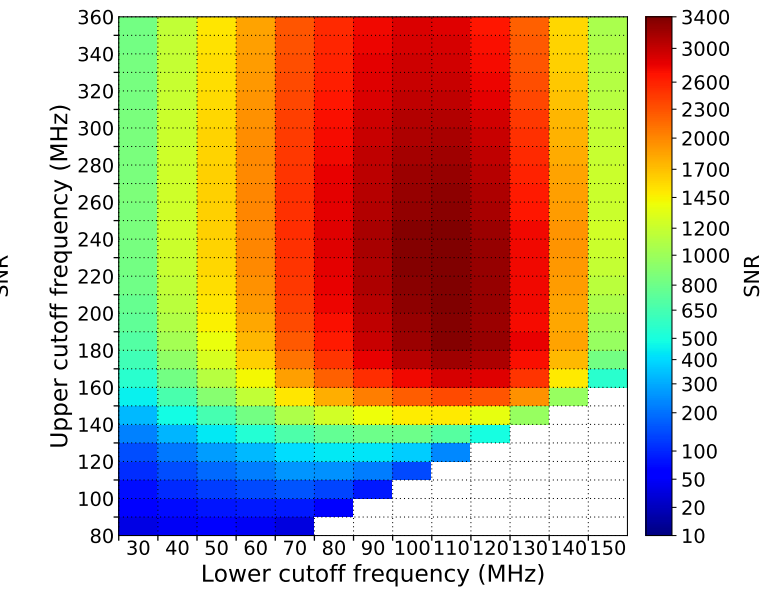

(b) IceTop: Thermal noise $=40 \mathrm{~K}$

Figure 2: Signal to noise ratio (SNR) obtained in different frequency bands for a $10 \mathrm{PeV} \gamma$-ray shower of $\theta=61^{\circ}$. The x-axis shows the lower cutoff frequency of the band and the y-axis shows the upper cutoff frequency. Each bin represents a frequency band. E.g. the lower left bin shows the band 30-80 MHz. Shown is the frequency scan for a station at the Cherenkov ring that has an off-axis angle of $1.1^{\circ}$ to the shower maximum and a core distance of $187 \mathrm{~m}$. The optimal band is seen to be 100-190 MHz. For lower thermal noise (b), the upper cutoff frequency can be extended to frequencies $>200 \mathrm{MHz}$ and we can still get a high level of SNR. Figures from [7] and [8].

With such an interplay of the signal and the noise, we can look at the parameter signal-tonoise ratio (SNR) defined in the time domain as SNR $=S^{2} / N^{2}$ where $S$ is the maximum of the signal envelope and $N$ is the rms noise. Such a parameter is looked at in different frequency bands of operation. This exercise, done for a station at the Cherenkov ring is shown in Figure 2, and reported in detail in references [7] and [8]. For $300 \mathrm{~K}$ thermal noise, it is seen that the SNR has a maximum value in the frequency band of 100-190 MHz. This is true at all locations of the 
shower footprint [7]. For a lower thermal noise (here $40 \mathrm{~K}$ ), the upper cut-off frequency can be extended even beyond $200 \mathrm{MHz}$ to obtain a high level of SNR. This is the case for the stations at the Cherenkov ring, and outside the Cherenkov ring. However, the station inside the Cherenkov ring has lower levels of SNR if the upper cut-off frequency is increased beyond $200 \mathrm{MHz}$ [8].

It was seen in this simulation study that the restriction to this frequency band of $100-190 \mathrm{MHz}$ improved the overall efficiency of the radio array. This was especially studied for the case of gamma ray and proton showers with a zenith angle of $61^{\circ}$ arriving at the South Pole. With this optimal frequency band the energy threshold can be lowered to $1.4 \mathrm{PeV}$ (with $100 \%$ efficiency for obtaining SNR $>10$ in 3 or more antennas), which is lower by an order of magnitude than what has been achieved so far [7]. The efficiency in the optimal band becomes better in the case where the thermal noise is $40 \mathrm{~K}$, which lowers the threshold further down to $1.1 \mathrm{PeV}$ [9]. Therefore such an optimum frequency band allows us to access lower energy ranges for the radio detection technique compared to what was possible until now. For the first time, this can allow us to search for PeV energy gamma rays coming from the Galactic Centre. The sensitivity for such a source for a radio array at the South Pole is given in Figure 3.

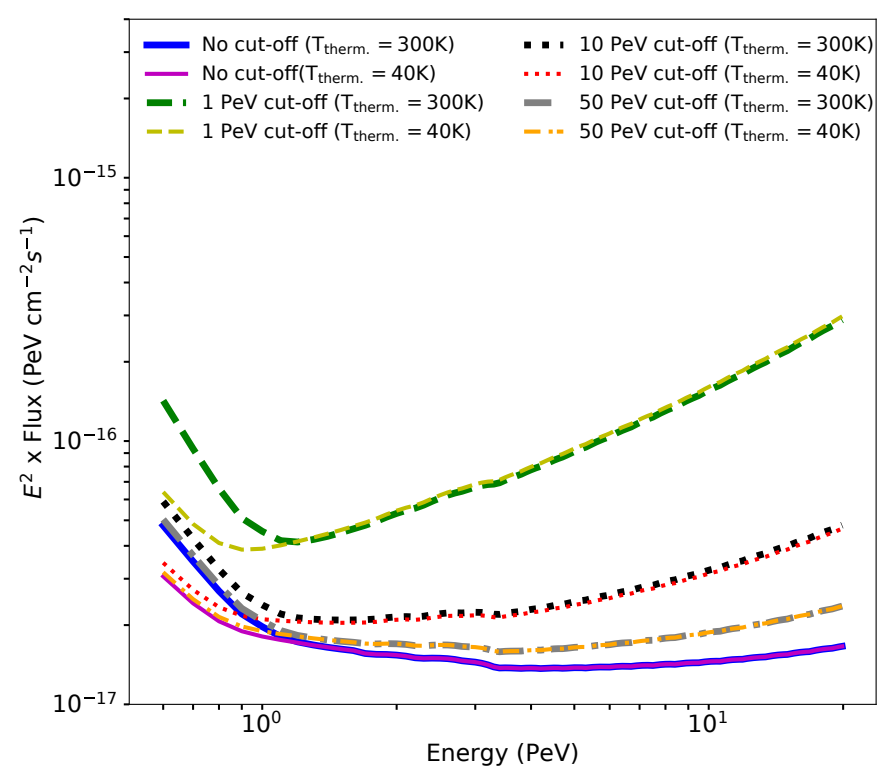

Figure 3: The integral sensitivity of a surface radio array at IceTop to a flux of gamma rays from the Galactic Centre, assuming various scenarios of the cut-off energy for a source that lies within a sky of $0.1^{\circ}$. The source flux is extrapolated from [13]. The shown integral sensitivity is for a period of 5 years for a gamma-hadron separation factor of 100. Also shown is the sensitivity when the radio array has a lower level of thermal noise [8].

The flux of the source is extrapolated from the observed gamma-ray flux of a candidate PeVatron at the centre of our Galaxy by the H.E.S.S. telescope [13]. The extrapolated flux to PeV energies included the absorption losses that arise due to the interaction of the incoming gamma rays with the photon field of the CMB. Also, different scenarios where the PeVatron has a cut-off in its spectrum are considered (eg. cutoff energy at $1 \mathrm{PeV}, 10 \mathrm{PeV}, 50 \mathrm{PeV}$ ). Assuming a gamma-hadron separation factor of 100 , the sensitivity to observe these gamma rays with $5 \sigma$ within a period of 5 
years, if they approach a frequency-optimised surface radio array at the South Pole, is shown for each possible source spectrum in Figure 3. Here, the cosmic ray flux measured by IceTop scaled to a sky of $0.1^{\circ}$ has been used as the background [8]. It is clear that such a measurement is possible only due to the frequency optimisation of this future radio array, which thereby improves the SNR and lowers the energy threshold.

The future surface enhancement of IceTop using radio antennas will therefore include this optimal frequency band [4]. Prototype antennas have been deployed at the South Pole which will be used to study the conditions at the South Pole [14]. These antennas operate in the frequency band of 50-350 MHz [12] which includes the optimum band of 100-190 MHz.

\section{Frequency optimisation for other locations}

In a manner similar to that done for IceTop, we can perform a frequency optimisation for radio signals from air showers that can be observed at other locations also. Here, such an optimisation done for the locations of GRAND and the Pierre Auger Observatory are reported. This is done in the light of the upcoming projects at these locations. GRAND will operate with the objective of measuring neutrinos and cosmic rays of the highest energies [15], while the radio upgrade of the Pierre Auger Observatory will attempt to detect ultra-high energy cosmic rays [16].

The simulations of radio signals from air showers that were used for the study reported in [17] were used for performing the frequency optimisation at the site of the Pierre Auger Observatory. These CoREAS simulations were generated with a magnetic field, observation level and atmosphere as that seen at the Pierre Auger Observatory, using a star shaped pattern for the antennas. The resulting scan of the SNR at various possible frequency bands of observation is shown in Figure $4 \mathrm{a}$. The same procedure as that followed for the study of the radio enhancement at IceTop is used here. This is for a station inside the Cherenkov ring (off-axis angle of $0.9^{\circ}$ from the shower maximum, core distance of $978 \mathrm{~m}$ and observation level $1564 \mathrm{~m}$ above the sea level), for a proton shower with an energy of $7.5 \mathrm{EeV}$ and arriving at the ground with a zenith angle of $80^{\circ}$. We see that for the location of the Auger Observatory also, the optimal band turns out to be $100-190 \mathrm{MHz}$, similar to what is obtained for IceTop. This is seen for the stations inside, outside and at the Cherenkov ring [8].

This result is quoted over here with the assumption that only the Galactic noise and the thermal noise of the antenna will be the major contributors to the total noise. However, this is not the case for the location of the Pierre Auger Observatory. A significant amount of man-made noise is reported at this location by the Auger Engineering Radio Array (AERA) within the band 30-80 MHz [18]. It is possible that this RFI noise level is more prominent in the optimal band 100-190 MHz seen in this study, when compared to the traditional frequency band of measurement (30-80 MHz). Within 30-80 MHz, AERA measures the overall behaviour of the noise as the Galactic noise, overridden by man-made noise peaks at certain frequencies. Therefore, a careful study of the noise at higher frequencies at the AERA site is needed to weigh the advantages of one band over the other.

For studying the frequency optimisation for GRAND, the simulations of the air showers were generated using ZHAires [19], with the environment (magnetic field, observation level) similar to that of the TREND location, in China [20]. Proton and iron initiated air showers with inclinations of $65^{\circ}-85^{\circ}$ with respect to the vertical, and neutrino generated showers that are horizontal or up- 
going were simulated. These showers were generated such that they were approaching an array of antennas with a spacing of $1 \mathrm{~km}$ between them, oriented along a slope within a range of of $5^{\circ}-20^{\circ}$.

The SNR was looked at in each possible frequency band of operation, for both cosmic ray and neutrino induced showers. This scan was performed similar to the manner done for IceTop, for stations inside, outside and at the Cherenkov ring. It was found in all cases, that the maximum SNR was obtained at a frequency band of 100-180 MHz, which is consistent with the results obtained for IceTop [15]. Figure 4b shows the SNR scan for an event from an upgoing neutrino producing a tau lepton that decays in flight and generates a shower, which is detected by an antenna array that lies on a mountain slope of $10^{\circ}$. A station that lies outside the Cherenkov ring with a distance of $\approx$ $11 \mathrm{~km}$ to the shower core, and has an off-axis angle of $1.9^{\circ}$ from the shower maximum is shown.

With this understanding of the optimal frequency band, it has been decided that GRAND will operate in the frequency range of 50-200 MHz [15]. Setting the lower cutoff frequency at $50 \mathrm{MHz}$ allows the inclusion of the well-studied lower frequencies in the measurements. With this, it will also be possible to cross-check with existing radio air-shower experiments, for consistency. From measurements with TREND, the predecessor of GRAND, it is understood that the level of manmade noise at Tianshan valley, the location of TREND, is very low [20]. Since such radio quiet environments will be available for GRAND also, it can be safely said that Galactic noise will be the main source of noise in this case. Therefore, the noise considered in this study is valid for conducting a frequency optimisation for GRAND. This will enable the enhancement of the SNR for GRAND with the optimal band, and thereby improve its performance.

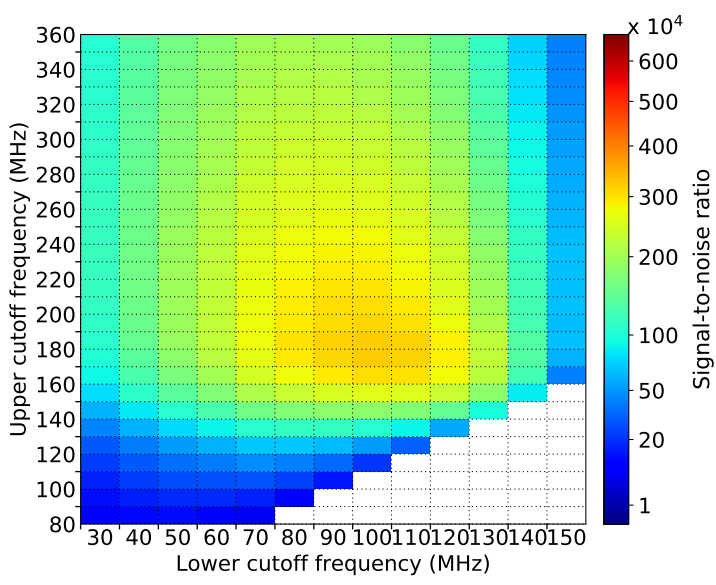

(a) Auger: inside the Cherenkov ring

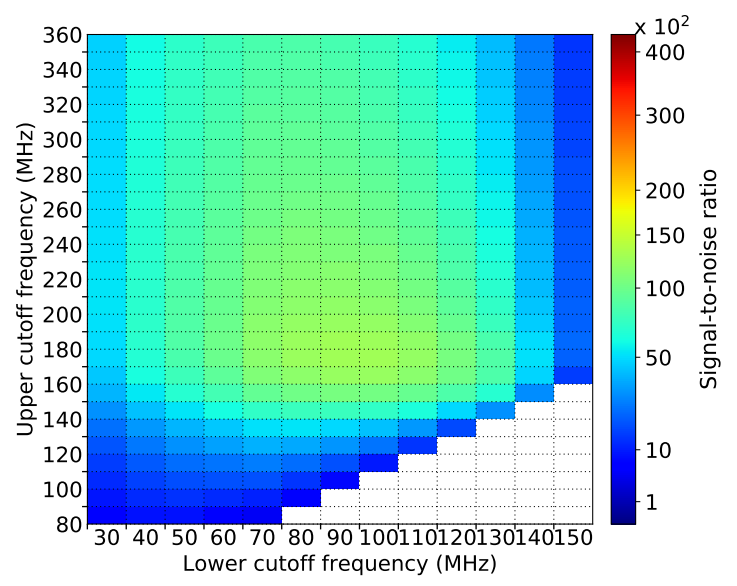

(b) GRAND: outside the Cherenkov ring

Figure 4: Signal-to-noise ratio at different frequency bands for showers simulated at AERA and GRAND. (a) Proton shower with energy 7.5 EeV and $\theta=80^{\circ}$. (b) An upgoing neutrino event with energy $0.5 \mathrm{EeV}$ that originates from $3^{\circ}$ below the horizon. Plots adapted from [8]. A noise temperature of $300 \mathrm{~K}$ is used for both cases. The maxima of the colour scales show the maximum values of the SNR that is obtained at the Cherenkov ring for these sample showers.

\section{Summary}

A frequency band optimisation performed for the radio enhancement of IceTop showed the optimum band to be 100-190 MHz. This optimisation done for the locations of GRAND and the 
Pierre Auger Observatory reveals the optimal band to be consistent with that obtained for IceTop. This implies that this optimum frequency band is universal and an operation in this band will improve the performance of all future radio arrays, especially for areas with low levels of RFI. This result is not surprising since the nature of radio emission is similar at all places. The only differences between the considered locations are the magnetic fields, the observation levels, and the local atmosphere. Furthermore, the same model of noise is used for studying the SNR at all locations. It has also been shown that ZHAires and CoREAS give consistent results for the observable radio emission [21]. Therefore, as expected, we obtain consistent results for the optimum frequency band at all the studied locations using the simulations shown here. The radio enhancement of IceTop plans to use antennas that operate in the frequency band $50-350 \mathrm{MHz}$, while the foreseen band of operation is $50-200 \mathrm{MHz}$ for GRAND. Both of these experiments will therefore include the optimal band, to which a digital filter can be applied.

Acknowledgements: We thank the members of the IceCube, the Pierre Auger, and the GRAND collaborations for their support. This work has partly made use of the Horizon Cluster hosted by Institut d'Astrophysique de Paris. A. Z. is supported by the APACHE grant (ANR-16-CE31-0001) of the French Agence Nationale de la Recherche.

\section{References}

[1] T. Huege, Phys.Rept. 620 (2016) 1-52

[2] F. G. Schröder, Prog.Part.Nucl.Phys. 93 (2017) 1-68

[3] R. Smida et al., Phys.Rev.Lett. 113 (2014) no.22, 221101

[4] F .G. Schröder for the IceCube-Gen2 Collaboration, arXiv:1811.00599

[5] R. Abbasi et al. (IceCube Collaboration), Nucl. Instrum. Methods A700, 188-220 (2013)

[6] M. G. Aartsen et al. (IceCube Collaboration), Journal of Instrumentation 12, P03012 (2017)

[7] A. Balagopal V., A. Haungs, T. Huege, F. G. Schröder, Eur. Phys. J. C 78, (2018) 111

[8] A. Balagopal V., PhD thesis (2019), DOI: 10.5445/IR/1000091377

[9] A. Balagopal V. et al., ARENA 2018, arXiv:1907.04171

[10] T. Huege, M. Ludwig, and C. W. James, AIP Conf. Proc., 1535:128, 2013

[11] H. V. Cane, MNRAS 189, 465-478 (1979)

[12] E. de Lera Acedo et al., Experimental Astronomy 39, 567 (2015)

[13] A. Abramowski et al. (H.E.S.S. Collaboration), Nature 531, 476 (2016)

[14] M. Renschler et al., J. Phys.: Conf. Ser. 1181 (2019) 012075

[15] J. Alvarez-Muñiz et al. (GRAND Collab.), Science China: Phys., Mech. \& Astron., 63, 219501 (2020)

[16] B. Pont for the Auger Collaboration, PoS(ICRC2019)395, this issue

[17] T. Huege, L. Brenk, and F. Schlüter, ARENA 2018, arXiv:1808.00729

[18] T. Huege and C. B. Welling, arXiv:1906.05148

[19] J. Alvarez-Muñiz, W. R. Carvalho, and E. Zas, Astroparticle Physics, 35, 325-341 (2012)

[20] D. Charrier et al. (TREND Collaboration), Astropart.Phys. 110 (2019) 15-29

[21] M. Gottowik et al., Astropart.Phys. 103 (2018) 87-93 